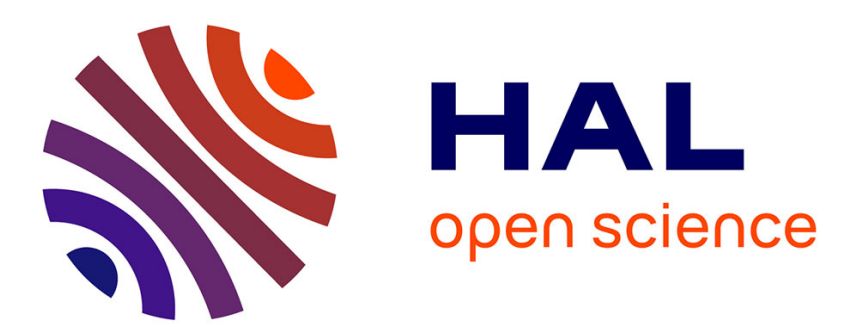

\title{
Control via state estimations of some nonlinear systems
}

Michel Fliess, Hebertt Sira-Ramirez

\section{To cite this version:}

Michel Fliess, Hebertt Sira-Ramirez. Control via state estimations of some nonlinear systems. IFAC Symposium on Nonlinear Control Systtems (NOLCOS 2004), 2004, Stuttgart, Germany. inria00001096

\section{HAL Id: inria-00001096 \\ https://hal.inria.fr/inria-00001096}

Submitted on 5 Feb 2006

HAL is a multi-disciplinary open access archive for the deposit and dissemination of scientific research documents, whether they are published or not. The documents may come from teaching and research institutions in France or abroad, or from public or private research centers.
L'archive ouverte pluridisciplinaire HAL, est destinée au dépôt et à la diffusion de documents scientifiques de niveau recherche, publiés ou non, émanant des établissements d'enseignement et de recherche français ou étrangers, des laboratoires publics ou privés. 


\title{
CONTROL VIA STATE ESTIMATIONS OF SOME NONLINEAR SYSTEMS
}

\author{
Michel Fliess ${ }^{*, 1}$ Hebertt Sira-Ramírez ${ }^{* *, 2}$ \\ * Laboratoire STIX, École polytechnique, 91128 Palaiseau, \\ France. \\ ** Cinvestav-IPN, Dept. Ing. Eléctrica Secc. Mecatrónica, \\ México D.F., México.
}

\begin{abstract}
We are proposing state estimators for nonlinear systems. Our techniques extend a previous work on state reconstructors for linear systems by the same authors (Reconstructeurs d'états, C.R. Acad. Sci. Paris, Série I, 338, 2004, 91-96), which bypasses some of the classic difficulties related to asymptotic observers and Kalman filtering (lack of robustness and knowledge of statistics). Our viewpoint, which avoids the integration of differential equations and therefore any asymptotic estimation, yields fast implementable algebraic formulae. Two concrete casestudies are presented, which are (differentially) flat. Our state estimation permits a state feedback control around the flatness-based reference trajectory. Convincing simulations are provided which demonstrate the robustness of our control strategy with respect to noises with unknown statistical properties.
\end{abstract}

Keywords: Nonlinear observability, flat systems, state estimation.

\section{INTRODUCTION}

Although the utilisation of (differentially) flat nonlinear systems (Fliess et al., 1995a, 1999) (see, also, Rudolph, 2003a, Sira-Ramírez \& Agrawal, 2004) has become quite widespread in industry, a fundamental question like the estimation of state variables is still far from being fully understood. This communication is proposing a clear cut solution which is extending the state reconstructors for linear systems obtained in Fliess \& Sira-Ramírez, 2004. Our approach, which does not necessitate

\footnotetext{
1 Partially supported by the action spécifique (CNRS, RTP 24) Méthodes algébriques pour les systèmes de communications numériques. The author belongs to the Équipe ALIEN of INRIA-Futurs. E-mail: Michel.Fliess@stix.polytechnique.fr

2 Partially supported by Conacyt-México under Research Project 42231-Y. The research was carried during a visit of the author to the Laboratory STIX of the École polytechnique. E-mail: hsira@mail.cinvestav.mx
}

the integration of any differential equations, is based on the estimation of the time derivatives of the (flat) output, up to some finite order. The formulae for those derivatives, which are quite different from those based on asymptotic observers (see, e.g., Ibrir, 2004) and from the classic ones in applied analysis (see, e.g., Lanczos, 1957), yield very fast calculations of the state. Our results are, like in Fliess \& Sira-Ramírez, 2003, 2004, quite robust with respect to perturbations ${ }^{3}$. Contrarily to the usual probabilistic setting, we do not need any precise statistical knowledge of the noise. We

\footnotetext{
3 Compare with the nonlinear estimation techniques based on numerical differentiation (see, e.g., Diop et al., 1994, 2000, 2001, Plestan et al., 1999, Röbenack, 2003). Although we will not try in such a conference paper to give any complete overview of the huge literature devoted to nonlinear state estimation, let us cite nevertheless the excellent book edited by Nijmeijer et al., 1999, which covers several recent trends in nonlinear asymptotic observers.
} 
can therefore handle noises of a rather arbitrary nature ${ }^{4}$.

Our paper is organised as follows. Section 2 is devoted to a brief overview of nonlinear system theory via differential algebra. Section 3 describes some salient features of our estimation procedure. Two illustrative examples are presented ${ }^{5}$ in section 4. Directions for future research are indicated in a short conclusion. We also discuss there some paradigms in nonlinear control.

Remark 1.1. The exact integral estimators of Fliess \& Sira-Ramírez, 2003, 2004, already permit to treat some particular nonlinear systems. See, e.g., Suarez-Castañon et al., 2003, for an application to Chua's systems, which are uncontrolled chaotic systems. The authors are able there to bypass celebrated techniques from dynamical system theory and control theory, such as Lyapunov's theory, Takens' approach, asymptotic observers, Kalman filtering, or adaptive control, which are difficult to implement.

\section{THE DIFFERENTIAL ALGEBRAIC APPROACH}

\subsection{Differential fields}

A differential field $K$ (see, e.g., Kolchin, 1973, Buium, 1994) will be here a commutative field ${ }^{6}$ of characteristic zero, which is equipped with a single derivation $\frac{d}{d t}: K \rightarrow K$ such that, for any $a, b \in K, \frac{d}{d t}(a+b)=\dot{a}+\dot{b}, \frac{d}{d t}(a b)=\dot{a} b+a \dot{b}$. A constant of $K$ is an element $c \in K$ such that $\dot{c}=0$. A (differential) field of constants is a differential field which only contains constants. The set of all constant elements of $K$ is a subfield, which is called the subfield of constants.

Notation 2.1. For any $a \in K, \nu=0,1,2, \ldots$, set $a^{(\nu)}=\frac{d^{\nu} a}{d t^{n} u}$.

A differential field extension $L / K$ is given by two differential fields $K, L$, such that the derivation of $K \subseteq L$ is the restriction to $K$ of the derivation of $L$. An element of $L$ is said to be differentially algebraic over $K$ if, and only if, it satisfies an algebraic differential equation with coefficients in $K$. It is said to be differentially transcendental if, and only

\footnotetext{
4 See Fliess, Mboup et al., 2003, Fliess, Join, Mboup \& Sira-Ramírez, 2004, Fliess, Join \& Sira-Ramírez, 2004, for applications of similar techniques to signal processing and linear fault diagnosis.

5 See Sira-Ramírez \& Fliess, 2004, for another example, and Fliess, Join \& Mounier, 2004, for an extension to nonlinear fault diagnosis.

${ }^{6}$ See, e.g., Atiyah et al., 1969, for basic field-theoretic notions.
}

if, it is not differentially algebraic. The extension $L / K$ is said to be differentially algebraic if, and only if, any element of $L$ is differentially algebraic over $K$. An extension which is not differentially algebraic is said to be differentially transcendental. A set $\left\{\xi_{\iota} \mid \iota \in I\right\}$ of elements in $L$ is said to be differentially algebraically independent over $K$ if, and only if, the set $\left\{\xi_{\iota}^{(\nu)} \mid \iota \in I, \nu \geq 0\right\}$ of derivatives of any order is algebraically independent over $K$. If a set is not differentially algebraically independent over $K$, it is differentially algebraically dependent over $K$. Such an independent set which is maximal with respect to inclusion is called a differential transcendence basis. The cardinalities of two such bases are equal. This cardinality is the differential transcendence degree of the extension $L / K$. Note that this degree is 0 if, and only if, $L / K$ is differentially algebraic.

Notation 2.2. Write $K\langle S\rangle$, where $S$ is a subset of $L$, the differential subfield of $L$ generated by $K$ and $S$.

\subsection{Nonlinear systems}

Let $k$ be a given differential ground field. A system ${ }^{7}$ is a finitely generated differential extension $K / k$. A dynamics is a system where a finite subset $\mathbf{u}=\left(u_{1}, \ldots, u_{m}\right) \subset K$ of control variables has been distinguished, such that the extension $K / k\langle\mathbf{u}\rangle$ is differentially algebraic. The control variables are said to be independent if, and only if, $\mathbf{u}$ is a differential transcendence basis of $K / k$. An input-output system is a dynamics where a finite subset $\mathbf{y}=\left(y_{1}, \ldots, y_{p}\right) \subset K$ of output variables has been distinguished.

Remark 2.1. The presentation of all these system theoretic notions may also be given in a differential geometric framework by utilising diffieties, i.e., prolongations and jets of infinite order (see, e.g., Fliess et al., 1999, and the references therein).

\subsection{Observability}

A system variable $\chi \in K$ is said to be observable (see Diop \& Fliess, 1991a, 1991b) if, and only if, it is algebraic over $k\langle\mathbf{u}, \mathbf{y}\rangle$. An input-output system $K / k$ is said to be observable if, and only if, the extension $K / k\langle\mathbf{u}, \mathbf{y}\rangle$ is algebraic.

Example 2.1. Set $k=\mathbf{R}$. Take the following state variable representation

$$
\begin{aligned}
& \dot{\mathbf{x}}=\mathbf{F}(\mathbf{x}, \mathbf{u}) \\
& \mathbf{y}=\mathbf{h}(\mathbf{x})
\end{aligned}
$$

\footnotetext{
7 See, e.g., Delaleau, 2002, Fliess et al., 1995a, Rudolph, 2003a, Sira-Ramírez \& Agrawal, 2004, for more details.
} 
where $\mathbf{x}=\left(x_{1}, \ldots, x_{n}\right)$ is the $n$-uple of state variables, $\mathbf{F}=\left(F_{1}, \ldots, F_{n}\right), \mathbf{h}=\left(h_{1}, \ldots, h_{p}\right)$ are respectively $n$-uples and $p$-uples of polynomial functions of their arguments. Our observability property means that any state variable is algebraic over $k\langle\mathbf{u}, \mathbf{y}\rangle$, i. e., is an algebraic function of the components of $\mathbf{u}, \mathbf{y}$ and of a finite number of their derivatives. It is known that this definition is equivalent to the classic observability rank condition (see, e.g., Isidori, 1989, Nijmeijer \& van der Schaft, 1990, Sastry, 1999) for system (1) (see Diop \& Fliess 1991a, 1991b, for full details).

\subsection{Flat systems}

A system $K / k$ is said to be (differentially) flat if, and only if, there exists a differential transcendence basis $\mathbf{z}=\left(z_{1}, \ldots, z_{m}\right)$ such that the algebraic closures of $K$ and $k\langle\mathbf{z}\rangle$ are the same. The set $\mathbf{z}$ is called a flat output. It means that

- any system variable is a function of the components of the flat outputs and of their derivatives up to some finite order,

- any component of the flat output is a function of the system variables and of their derivatives up to some finite order.

The next properties are easy:

Proposition 2.1. Take a flat dynamics with independent control variables, then the cardinalities of $\mathbf{z}$ and $\mathbf{u}$ are equal.

Proposition 2.2. Assume that system (1) is flat and that $\mathbf{y}$ is a flat output. System (1) is then observable.

\section{ESTIMATING DERIVATIVES}

\subsection{Polynomial time signals ${ }^{8}$}

Consider the polynomial $x_{N}(t)=\sum_{\nu=0}^{N} x^{(\nu)}(0) \frac{t^{\nu}}{\nu !}$ $\in \mathbf{R}[t]$ of degree $N$. The usual rules of symbolic calculus yield in distributions theory (Schwartz, 1966)

$$
x_{N}^{(N+1)}(t)=x_{N}(0) \delta^{(N)}+\ldots+x_{N}^{(N)}(0) \delta
$$

where $\delta$ is the Dirac measure at 0 . From $t \delta=$ $0, t \delta^{(\alpha)}=-\alpha \delta^{(\alpha-1)}, \alpha \geq 1$, we obtain the following triangular system of linear equations

8 See Fliess, Mboup et al., 2003, Fliess, Join, Mboup \& Sira-Ramírez, 2004, for a presentation via operational calculus, which we believe to be clearer. for determining the values ${ }^{9}$ of the derivatives ${ }^{10}$ $x_{N}^{(\nu)}(0)$ :

$$
\begin{aligned}
& t^{\alpha} x_{N}^{(N+1)}(t)=-t^{\alpha}\left(x_{N}(0) \delta^{(N)}\right. \\
& \left.+\dot{x}_{N}(0) \delta^{(N-1)}+\ldots+x_{N}^{(N)}(0) \delta\right) \\
& \alpha=0, \ldots, N
\end{aligned}
$$

The time derivatives of $x(t), t \neq 0$, are removed by integrating with respect to time both sides of equation (2) at least $N$ times:

$$
\begin{aligned}
& \int_{1}^{(\nu)} \tau_{1}^{\alpha} x_{N}^{(N+1)}\left(\tau_{1}\right)=-\int^{(\nu)} \tau_{1}^{\alpha}\left(x_{N}(0) \delta^{(N)}+\right. \\
& \left.\dot{x}_{N}(0) \delta^{(N-1)}+\ldots+x_{N}^{(N)}(0) \delta\right) \\
& \nu \geq N
\end{aligned}
$$

where $\int^{(\nu)}=\int_{0}^{t} \int_{0}^{\tau_{\nu-1}} \ldots \int_{0}^{\tau_{1}}$.

\subsection{General time signals}

Consider a real-valued time function $x(t)$ which is assumed to be analytic around $t=0$. Introduce its truncated Taylor expansion

$$
x(t)=\sum_{\nu=0}^{N} x^{(\nu)}(0) \frac{t^{\nu}}{\nu !}+O\left(t^{\nu+1}\right)
$$

Approximate $x(t)$ in the interval $(0, \varepsilon), \varepsilon>0$, by a polynomial $x_{N}(t)=\sum_{\nu=0}^{N} x^{(\nu)}(0) \frac{t^{\nu}}{\nu !}$ of degree $N$. A "good" estimate ${ }^{11}$ of the first derivatives is obtained by replacing $x_{N}(t)$ in the left hand sides of equations (2) and (3) by $x(t)$.

\subsection{Some practical rules}

Our estimations of the flat output time derivatives and of the states are based on the following practical rules:

(1) We adopt a sufficiently high order time polynomial approximation of the output signals

(2) "High frequency" perturbations are attenuated by utilising adequate low pass filters like elementary iterated time integrals. Contrarily to the usual probabilistic framework, we do not need any precise statistical knowledge of the noises. See Fliess \& Sira-Ramírez, 2003, 2004, Fliess, Mboup et al., 2003, for more details.

(3) We are periodically resetting the calculations as the adopted time approximation becomes non-valid.

\footnotetext{
9 The multiplication by $-t$ corresponds in operational calculus to the algebraic derivative $\frac{d}{d s}$ (see Fliess \& SiraRamírez, 2003, and the references therein).

${ }^{10}$ Those quantities are linearly identifiable (see Fliess \& Sira-Ramírez, 2003, Fliess, Mboup et al., 2003).

${ }^{11}$ See Fliess, Join, Mboup \& Sira-Ramírez, 2004, for more details.
} 


\section{TWO CONCRETE CASE STUDIES}

Remark 4.1. Some of the system equations below are not differentially algebraic since they involve sine or cosine functions of a system variable $\chi$. It is nevertheless possible to recover the above formalism by introducing $\tan \frac{\chi}{2}$, as in Fliess et al., 1995a. Our mathematical formalism is therefore applicable.

\subsection{Control of a unicycle ${ }^{12}$}

The following kinematic model is flat

$$
\dot{x}=u_{1} \cos \theta, \quad \dot{y}=u_{1} \sin \theta, \quad \dot{\theta}=u_{2}
$$

It represents a unicycle contained on a vertical plane and rolling on a horizontal coordinate plane $(x, y)$ with orientation angle $\theta$ with respect to the $x$-axis. We are measuring the coordinates $(x, y)$ of the flat output, which is the wheel point of contact with the plane. A first order extension of the control input $u_{1}$ is required by the trajectory tracking dynamic feedback linearising controller

$$
\begin{gathered}
\left(\begin{array}{c}
\dot{u}_{1} \\
u_{2}
\end{array}\right)=\left(\begin{array}{cc}
\frac{[\dot{x}]_{e}}{\sqrt{\left([\dot{x}]_{e}\right)^{2}+\left([\dot{y}]_{e}\right)^{2}}} & \frac{[\dot{y}]_{e}}{\sqrt{\left([\dot{x}]_{e}\right)^{2}+\left([\dot{y}]_{e}\right)^{2}}} \\
-\frac{[\dot{y}]_{e}}{\left([\dot{x}]_{e}\right)^{2}+\left([\dot{y}]_{e}\right)^{2}} & \frac{[\dot{x}]_{e}}{\left([\dot{x}]_{e}\right)^{2}+\left([\dot{y}]_{e}\right)^{2}}
\end{array}\right) \\
\times\left(\begin{array}{c}
\ddot{x}^{*}(t)-2 \xi \omega_{n}\left([\dot{x}]_{e}-\dot{x}^{*}(t)\right)-\omega_{n}^{2}\left(x-x^{*}(t)\right) \\
\ddot{y}^{*}(t)-2 \xi \omega_{n}\left([\dot{y}]_{e}-\dot{y}^{*}(t)\right)-\omega_{n}^{2}\left(y-y^{*}(t)\right)
\end{array}\right)
\end{gathered}
$$

where $x^{*}(t), y^{*}(t)$ represent a desired flatnessbased trajectory. Following section 3 the velocity estimates $[\dot{x}]_{e},[\dot{y}]_{e}$ are given by

$$
\begin{aligned}
& {[\dot{x}(t)]_{e} }=\frac{1}{\left(t-t_{i}\right)^{3}}\left[6\left(t-t_{i}\right)^{2} x(t)\right. \\
&\left.-18 \int_{t_{i}}^{t}\left(\sigma-t_{i}\right) x(\sigma) d \sigma+6 \int_{t_{i}}^{t} \int_{t_{i}}^{\sigma} x(\lambda) d \lambda d \sigma\right] \\
& {[\dot{y}(t)]_{e}=\frac{1}{\left(t-t_{i}\right)^{3}}\left[6\left(t-t_{i}\right)^{2} y(t)\right.} \\
&\left.-18 \int_{t_{i}}^{t}\left(\sigma-t_{i}\right) y(\sigma) d \sigma+6 \int_{t_{i}}^{t} \int_{t_{i}}^{\sigma} y(\lambda) d \lambda d \sigma\right]
\end{aligned}
$$

During the re-initialisation intervals, $\left[t_{i}, t_{i}+\delta\right]$, we set the estimated derivatives to take $a$ constant value, represented by the corresponding derivative value at time $t_{i}^{-}$, i.e., at the end of the preceding estimation interval $\left[t_{i-1}, t_{i}\right]$. The simulations below depict the response of the kinematic model of the car. We have chosen here to track, in a counterclockwise sense, a circular trajectory of radius equal to one, with angular velocity $\omega=0.15$ $[\mathrm{rad} / \mathrm{s}]$. The controller parameters were set to be

12 This simplified model of unicycle has often been studied in the literature (see, e.g., Sira-Ramírez \& Agrawal, 2004). $\xi=0.81, \omega_{n}=0.7[\mathrm{rad} / \mathrm{s}]$. The small interval of time $\delta$, after each re-initialisation instant, prescribed for allowing the numerical processor to obtain a precise quotient calculation, was taken to be $\delta=0.05[\mathrm{~s}]$. The spikes in the control are largely due to those resettings.

Let $\operatorname{rect}(t)=\operatorname{comp}(t)-0.5$ be a zero mean random process, where $\operatorname{comp}(t)$ is a computer generated random processes ${ }^{13}$ synthesised on the basis of a piecewise constant random variable uniformly distributed in the interval $[0,1]$. Set $\eta_{1}=0.01 \operatorname{rect}(t), \eta_{2}=0.01 \operatorname{rect}(t), \eta_{3}=$ $-0.01 \operatorname{rect}(t)$, and $\nu_{1}(t)=0.0001 \operatorname{rect}(t), \nu_{2}(t)=$ $0.0002 \operatorname{rect}(t)$. The robustness was tested with

$$
\begin{aligned}
& \dot{z}=u_{1} \cos \theta+\eta_{1}(t) \\
& \dot{q}=u_{1} \sin \theta+\eta_{2}(t) \\
& \dot{\theta}=u_{2}+\eta_{3}(t) \\
& x=z+\nu_{1}(t) \\
& y=q+\nu_{2}(t)
\end{aligned}
$$

\subsection{Control of a dc motor pendulum}

Consider, following Fan \& Arcak, 2003, a flat system ${ }^{14}$ consisting of a DC-motor joined to an inverted pendulum through a torsional spring

$$
\begin{aligned}
J_{m} \ddot{\theta}_{m} & =\kappa\left(\theta_{l}-\theta_{m}\right)-B \dot{\theta}_{m}+K_{\tau} u \\
J_{l} \ddot{\theta}_{l} & =-\kappa\left(\theta_{l}-\theta_{m}\right)-m g h \sin \left(\theta_{l}\right) \\
y & =\theta_{l}
\end{aligned}
$$

$\theta_{m}$ represents the angular deviation of the motor shaft. The flat output $y=\theta_{l}$, where $\theta_{l}$ is the inverted pendulum angular position, is measured. Asymptotic tracking of a given smooth reference trajectory $y^{*}(t)=\theta_{l}^{*}(t)$ is achieved by the feedback controller

$$
\begin{aligned}
u= & \frac{1}{K_{\tau}}\left\{\frac { J _ { m } } { \kappa } \left[J_{l} v+\kappa \ddot{y}_{e}\right.\right. \\
& \left.+m g h\left(\ddot{y}_{e} \cos y-\left(\dot{y}_{e}\right)^{2} \sin (y)\right)\right] \\
& +J_{l} \ddot{y}_{e}+m g h \sin (y) \\
& \left.+\frac{B}{\kappa}\left[J_{l} y_{e}^{(3)}+\kappa \dot{y}_{e}+m g h \dot{y}_{e} \cos y\right]\right\} \\
v= & {\left[y^{*}(t)\right]^{(4)}-\gamma_{4}\left(y_{e}^{(3)}-\left[y^{*}(t)\right]^{(3)}\right) } \\
& -\gamma_{3}\left(\ddot{y}_{e}-\ddot{y}^{*}(t)\right)-\gamma_{2}\left(\dot{y}_{e}-\dot{y}^{*}(t)\right) \\
& -\gamma_{1}\left(y-y^{*}(t)\right)
\end{aligned}
$$

where the subscript " $e$ " denotes the estimated value of the derivative. The design parameters $\gamma_{1}, \cdots, \gamma_{4}$ are chosen so that the resulting characteristic polynomial is Hurwitz. The availability of $y=\theta_{l}$ and of its first three time derivatives $\dot{y}, \ddot{y}$, $y^{(3)}$ readily allows one to implement the proposed

\footnotetext{
${ }^{13}$ The noises here and in the next example are not usual in the traditional mathematical theory of nonlinear filtering. ${ }^{14}$ This single input system is of course static state feedback linearisable.
} 

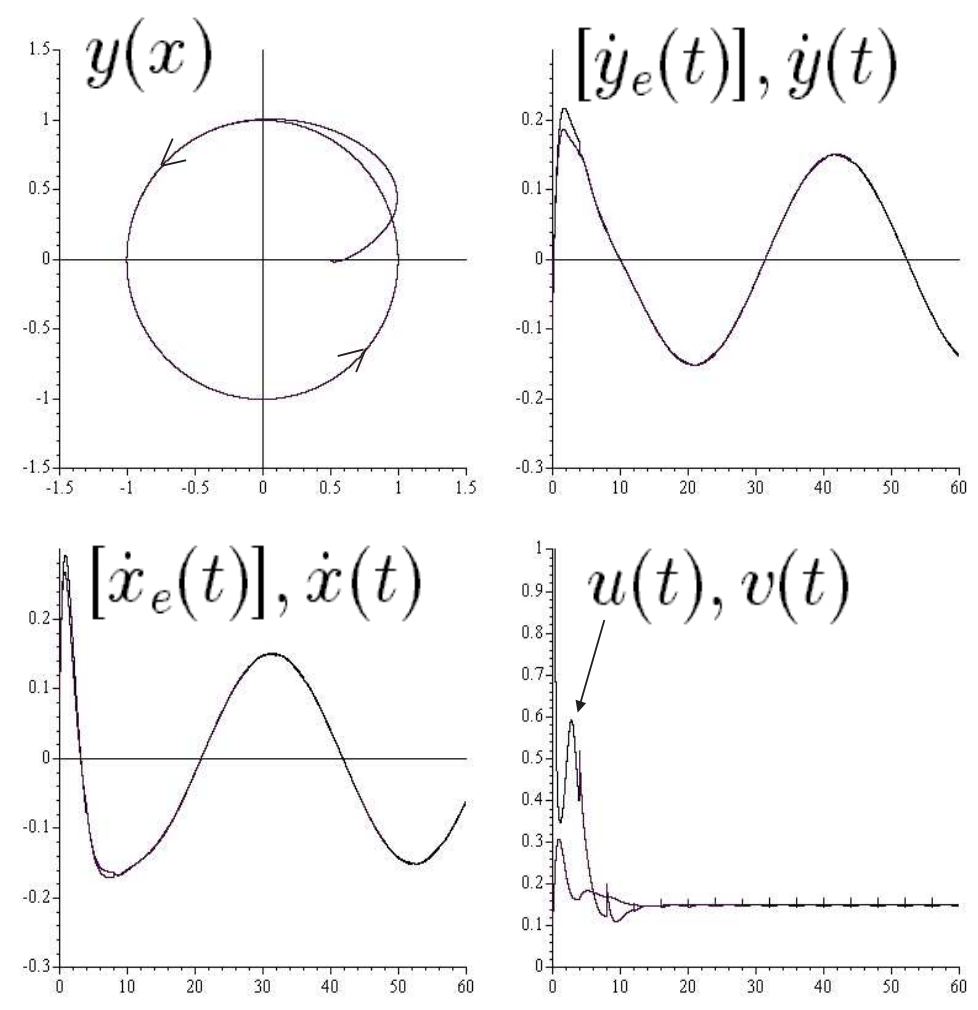

Fig. 1. Closed loop response of the unicycle model to dynamic feedback control based on the algebraic velocities estimations.
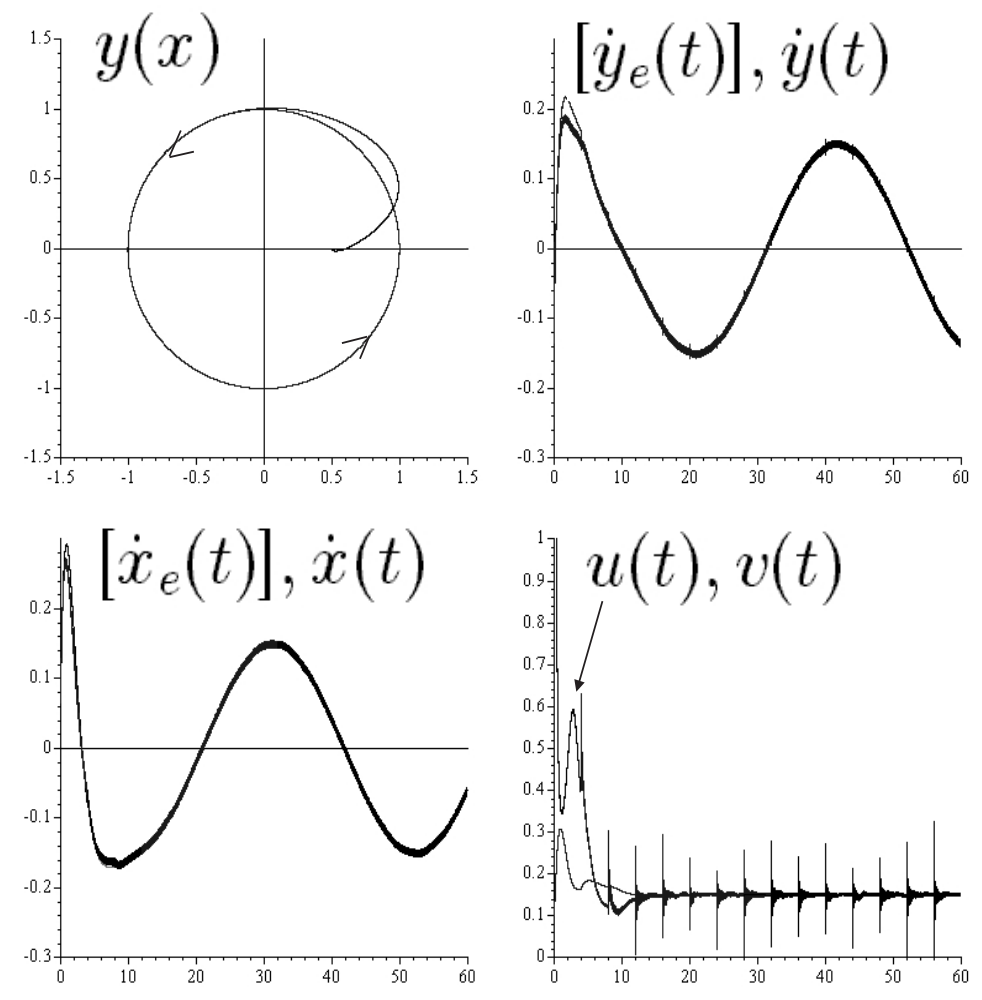

Fig. 2. Closed loop response of the perturbed unicycle model to dynamic feedback control based on the algebraic velocities estimations with noisy measurements. 
flatness based trajectory tracking feedback control law. Note that the unmeasured motor shaft angular position would be estimated as

$$
\widehat{\theta}_{m}=\frac{1}{\kappa}\left[J_{l} \ddot{y}_{e}+\kappa y+m g h \sin y\right]
$$

Similar computations to those with the first example yield

$$
\begin{aligned}
& \dot{y}_{e}(t)= \\
& \frac{1}{\left(t-t_{i}\right)^{7}}\left[42\left(t-t_{i}\right)^{6} y-882\left(\int_{t_{i}}\left(t-t_{i}\right)^{5} y\right)\right. \\
& +7350\left(\int_{t_{i}}^{(2)}\left(t-t_{i}\right)^{4} y\right) \\
& -29400\left(\int_{t_{i}}^{(3)}\left(t-t_{i}\right)^{3} y\right) \\
& +52920\left(\int_{t_{i}}^{(4)}\left(t-t_{i}\right)^{2} y\right)-35280\left(\int_{t_{i}}^{(5)}\left(t-t_{i}\right) y\right) \\
& \left.+5040\left(\int_{t_{i}}^{(6)} y\right)\right] \\
& \ddot{y}_{e}(t)= \\
& \frac{1}{\left(t-t_{i}\right)^{7}}\left[35\left(t-t_{i}\right)^{6} \dot{y}_{e}-630\left(t-t_{i}\right)^{5} y\right. \\
& +7350\left(\int_{t_{i}}\left(t-t_{i}\right)^{(4)} y\right)-29400\left(\int_{t_{i}}^{(2)}\left(t-t_{i}\right)^{3} y\right) \\
& +52920\left(\int_{t_{i}}^{(3)}\left(t-t_{i}\right)^{2} y\right)-35280\left(\int_{t_{i}}^{(4)}\left(t-t_{i}\right) y\right) \\
& \left.+5040\left(\int_{t_{i}}^{(5)} y\right)\right] \\
& y_{e}^{(3)}(t)= \\
& \frac{1}{\left(t-t_{i}\right)^{7}}\left[28\left(t-t_{i}\right)^{6} \ddot{y}_{e}-420\left(t-t_{i}\right)^{5} \dot{y}_{e}\right. \\
& +4200\left(t-t_{i}\right)^{4} y-29400\left(\int_{t_{i}}\left(t-t_{i}\right)^{3} y\right) \\
& +52920\left(\int_{t_{i}}^{(2)}\left(t-t_{i}\right)^{2} y\right)-35280\left(\int_{t_{i}}^{(3)}\left(t-t_{i}\right) y\right) \\
& \left.+5040\left(\int_{t_{i}}^{(4)} y\right)\right]
\end{aligned}
$$

Figure 3 depicts the closed loop performance, in a rest-to-rest trajectory tracking task, when the flatness based controller is synthesised on the basis of the estimated derivatives of the flat output. The estimated motor shaft angular position $\widehat{\theta}_{m}$ is also shown. The controller parameters were set so that the characteristic polynomial of the closed loop linearised system coincided with $\left(s^{2}+2 \zeta \omega_{n} s+\right.$ $\left.\omega_{n}^{2}\right)^{2}, \zeta=0.81, \omega_{n}=7.5[\mathrm{rad} / \mathrm{s}]$. The parameter $\epsilon$ representing the interval in which we block the singularity off the calculation was chosen to be $\epsilon=0.04[\mathrm{~s}]$. The estimation period $T$ for the derivatives calculations was chosen as $T=0.3[\mathrm{~s}]$. The desired rest to rest maneuver for the pendulum angular position takes $\theta_{l}$ from $\theta_{l}\left(t_{\text {init }}\right)=0$ [rad], with $t_{\text {init }}=0.5[\mathrm{~s}]$ to $\theta_{l}\left(t_{\text {final }}\right)=1.5[\mathrm{rad}]$, with $t_{\text {final }}=1.5[\mathrm{~s}]$. The jumps in the figure are due to resettings and to the adoption of a constant value while the initial singularity is overcome. To test the robustness we have included an additive perturbation term in the output measurement equation $y=\theta_{l}+a \eta(t)$, where $\eta(t)$ is a computer generated piecewise constant noise comprised of a sequence of pseudo random variables exhibiting an uniform probability distribution in the interval $[-0.5,0.5][\mathrm{rad}]$, at each small integration step of value $10^{-3}[\mathrm{~s}]$. The amplitude of the noise was chosen to be $a=0.5 \times 10^{-5}$ [rad]. Figure 4 depicts the computer simulations.

\section{CONCLUSION}

The extension to state estimation when the measured output is non-flat and/or for non-flat systems is straightforward. Identification of unknown parameters will be done in future publications along the same lines ${ }^{15}$. We will also achieve, as in Fliess \& Sira-Ramírez, 2003, for linear systems, nonlinear closed loop identification.

This major progress in nonlinear system theory is made possible by a complete change of viewpoint $^{16}$ and certainly not by solving the longstanding mathematical problems related to nonlinear asymptotic observers or nonlinear Kalman filtering:

(1) Estimated values are no more given in an asymptotic way with respect to a large time ${ }^{17}$. On the contrary the exact values of the unknown quantities are obtained when $t \rightarrow 0$, i.e., for very short time windows.

(2) Those estimated values result from algebraic formulae which are providing good approximations in real time even in a noisy environment.

\footnotetext{
${ }^{15}$ See Diop \& Fliess, 1991a, 1991b, for an algebraic definition of nonlinear identifiability: An unknown parameter is identifiable if, and only if, it is a function of the control and output variables and their time derivatives, up to some finite order.

${ }^{16}$ Flatness may also be viewed as a new understanding of the controllability concept (see Fliess, 2000). Note that the celebrated state variable representation becomes also much less crucial.

${ }^{17}$ Remember that the feedback stabilisation of a nonholonomic vehicle around a rest point was also given in a non asymptotic manner by Fliess et al., 1995b. This feedback synthesis has often been most successfully implemented in practice.
} 

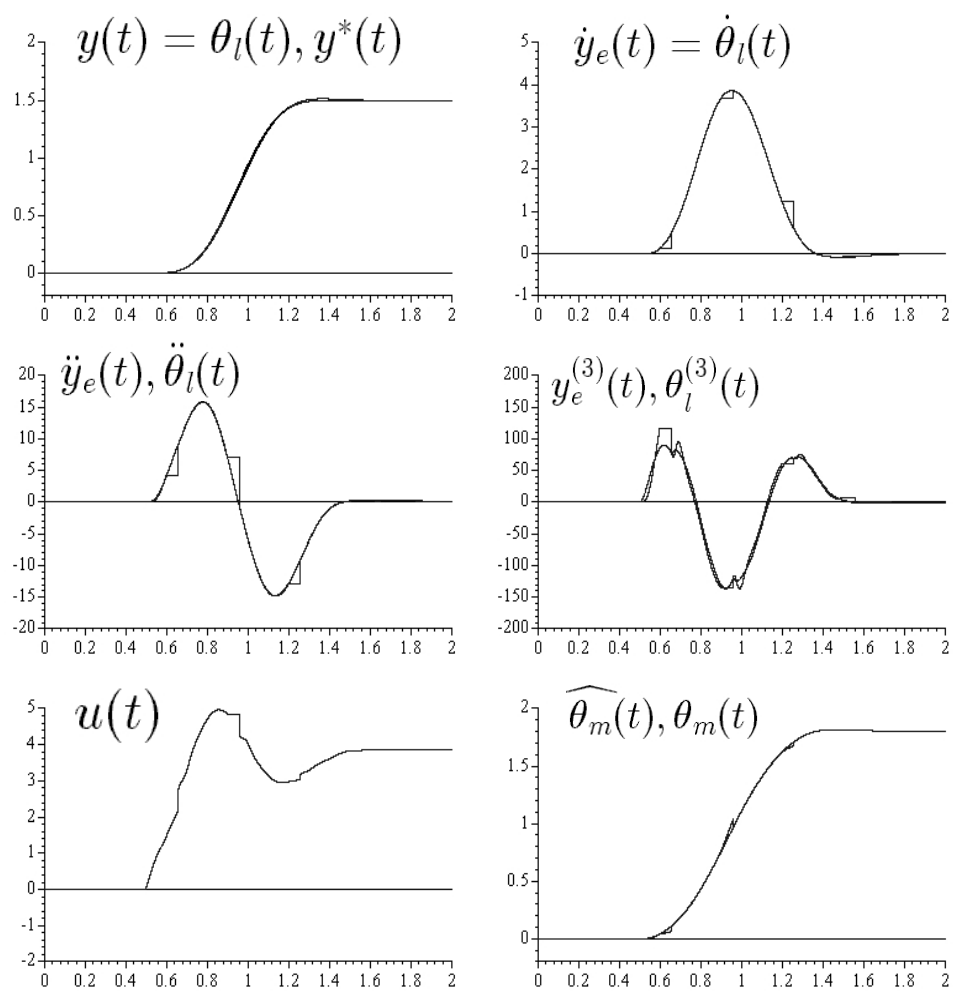

Fig. 3. Closed loop system response to feedback control based on the estimations of piecewise continuous flat output derivatives.
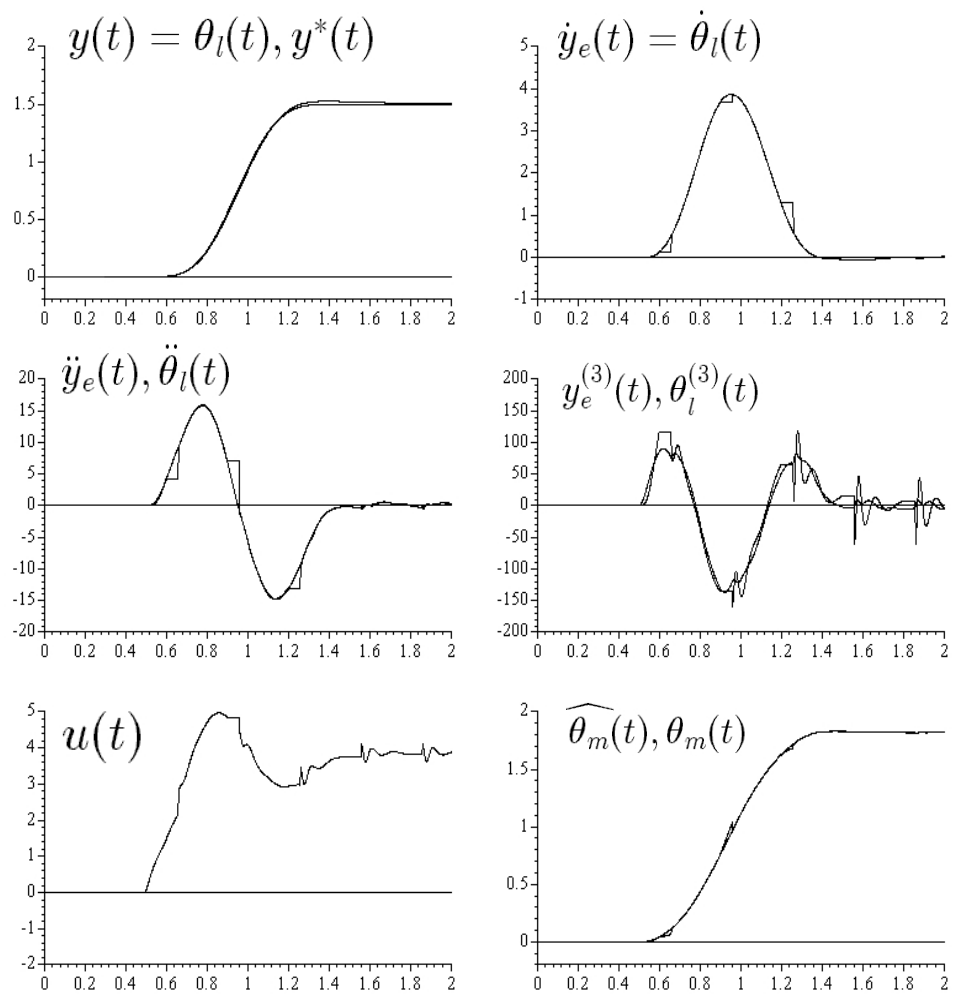

Fig. 4. Closed loop system response to feedback control based on the estimations of piecewise continuous flat output derivatives obtained with noisy output measurements. 
(3) We do not need any probabilistic machinery ${ }^{18}$. The noises are viewed as high frequency perturbations which may be attenuated by low pass filters without any precise knowledge of their statistical properties (see, also, Fliess \& Sira-Ramírez, 2003, 2004, Fliess, Mboup et al., 2003, Fliess, Join \& Sira-Ramírez, 2004).

(4) We do not need any discretisation scheme of the original system ${ }^{19}$ nor of a complex asymptotic observer. Our numerical techniques, which will be further developed, are of a complete different nature.

\section{REFERENCES}

M. Atiyah, I. McDonald, 1969, Introduction to Commutative Algebra, Addison-Wesley, Reading, MA.

A. Buium, 1994, Differential Algebra and Diophantine Geometry, Hermann, Paris.

E. Delaleau, 2002, "Algèbre différentielle", in Mathématiques pour les systèmes dynamiques, J.P. Richard, Ed., vol. 2, chap. 6, pp. 245-268, Hermès, Paris.

S. Diop, M. Fliess, 1991a, "On nonlinear observability", Proc. $1^{\text {st }}$ Europ. Control Conf., Hermès, Paris, pp. 152157.

S. Diop, M. Fliess, 1991b, "Nonlinear observability, identifiability and persistent trajectories", Proc. $36^{\text {th }}$ IEEE Conf. Decision Control, Brighton, pp. 714-719.

S. Diop, V. Fromion, J.W. Grizzle, 2001, "A global exponential observer based on numerical differentiation", Proc. $40^{\text {th }}$ Conf. Decision Control, Orlando, FL.

S. Diop, J.W. Grizzle, F. Chaplais, 2000, "On numerical differentiation for nonlinear estimation", Proc. $39^{\text {th }}$ Conf. Decision Control, Sidney.

S. Diop, J.W. Grizzle, P.E. Moraal, A. Stefanopoulou, 1994, "Interpolation and numerical differentiation for observer design", Proc. Amer. Control Conf., Baltimore, pp. 1329-1333.

X. Fan, M. Arcak, 2003, "Observer design for systems with multivariable monotone nonlinearities", Systems Control Lett., 50, 319-330.

M. Fliess, 2000, "Variations sur la notion de contrôlabilité", in Journée Soc. math. France, Paris, pp. 47-86.

M. Fliess, C. Join, M. Mboup, H. Sira-Ramírez, 2004, "Compression différentielle de transitoires bruités", to appear.

M. Fliess, C. Join, H. Mounier, 2004, "An introduction to nonlinear fault diagnosis with an application to a congested internet router", in Advances in Communication Control Networks, C.T. Abdallah, J. Chiasson, S. Tabouriech, Eds, Lect. Notes Control Inform. Sci., Springer, London.

M. Fliess, C. Join, H. Sira-Ramírez, 2004, "Robust residual generation for linear fault diagnosis: an algebraic setting with examples", Internat. J. Control, to appear.

\footnotetext{
${ }^{18}$ See, e.g., Jaulin et al., 2001, for another approach to estimation without probability.

${ }^{19}$ Let us recall that flatness-based control does not necessitate for its practical implementation any discretisation of the system (see, e.g., Rudolph, 2003a, Sira-Ramírez \& Agrawal, 2004, and the references therein). This applies also to the flatness-based control of infinite-dimensional systems, like delay systems or systems with distributed parameters (see, e.g., Rudolph, 2003a, 2003b, Rudolph et al., 2003, Sira-Ramírez \& Agrawal, 2004, and the references therein).
}

M. Fliess, J. Lévine, P. Martin, P. Rouchon, 1995a, "Flatness and defect of non-linear systems: introductory theory and examples", Internat. J. Control, 61, 1327-1361.

M. Fliess, J. Lévine, P. Martin, P. Rouchon, 1995b, "Design of trajectory stabilizing feedback for driftless flat systems", Proc. $3^{\text {rd }}$ Europ. Control Conf., Rome, pp. $1882-1887$.

M. Fliess, J. Lévine, P. Martin, P. Rouchon, 1999, "A Lie-Bäcklund approach to equivalence and flatness of nonlinear systems", IEEE Trans. Automat. Control, 44, 922-937.

M. Fliess, M. Mboup, H. Mounier, H. Sira-Ramírez, 2003, "Questioning some paradigms of signal processing via concrete examples", in Algebraic Methods in Flatness, Signal Processing and State Estimation, H. SiraRamírez, G. Silva-Navarro, Eds., pp. 1-21, Editiorial Lagares, México.

M. Fliess, H. Sira-Ramírez, 2003, "An algebraic framework for linear identification", ESAIM Control Optim. Calc. Variat., 9, 151-168.

M. Fliess, H. Sira-Ramírez, 2004, "Reconstructeurs d'états", C.R. Acad. Sci. Paris, Série I, 338, 91-96.

S. Ibrir, 2004, "Linear time-derivatives trackers", Automatica, 40, 397-405.

A. Isidori, 1989, Nonlinear Control Systems, $2^{\text {nd }}$ ed., Springer, New York.

L. Jaulin, M. Kieffer, O. Didrit, E. Walter, 2001, Applied Interval Analysis, with Examples in Parameter and State Estimation, Robust Control, and Robotics, Springer, London.

E.R. Kolchin, 1973, Differential Algebra and Algebraic Groups, Academic Press, New York.

C. Lanczos, 1957, Applied Analysis, Prentice Hall, Englewood Cliffs, NJ.

H. Nijmeijer, T.I. Fossen, Eds, 1999, New Directions in Nonlinear Observer Design, Lect. Notes Control Informat. Sci. 244, Springer, London.

H. Nijmeijer, A.J. van der Schaft, 1990, Nonlinear Dynamical Control Systems, Springer, New York.

F. Plestan, J.W. Grizzle, 1999, "Synthesis of nonlinear observers via structural analysis and numerical differentiation", Proc. $5^{\text {th }}$ Europ. Control Conf., Karlsruhe.

K. Röbenack, 2003, Beobachterentwurf für nichtlineare Zustandssysteme mit Hilfe des Automatischen Differenzierens, Shaker Verlag, Aaachen.

J. Rudolph, 2003a, Beiträge zur flacheitsbasierten Folgeregelung linearer und nichtlinearer Syteme endlicher und undendlicher Dimension, Shaker Verlag, Aachen.

J. Rudolph, 2003b, Flatness Based Control of Distributed Parameter Control, Shaker Verlag, Aaachen.

J. Rudolph, J. Winkler, F. Woittennek, 2003, Flatness Based Control of Distributed Parameter Systems: Computer Exercises from Various Technological Domains, Shaker Verlag, Aachen.

S. Sastry, 1999, Nonlinear Systems - Analysis, Stability, and Control, Springer, New York.

L. Schwartz, 1966, Théorie des distributions, $2^{\text {nd }}$ ed., Hermann, Paris.

H. Sira-Ramírez, S. Agrawal, 2004, Differentially Flat Systems, Marcel Dekker, New York.

H. Sira-Ramírez, M. Fliess, 2004, "On the output feedback control of a synchronous generator", Proc. $43^{\text {rd }}$ IEEE Conf. Decision Control, Atlantis, Bahamas.

M.S. Suarez-Castañon, C. Aguilar-Ibáñez, F. Flores-Ando, 2003, "Reconstructing the states and parameters of Chua's system based on successive integrations of the output", Physics Lett. A, 317, 265-274. 